\title{
Functional characterization of Rorippa indica defensin and its efficacy against Lipaphis erysimi
}

\author{
Poulami Sarkar ${ }^{1}$, Jagannath Jana², Subhrangshu Chatterjee ${ }^{2}$ and Samir Ranjan Sikdar ${ }^{\text {* }}$
}

\begin{abstract}
Rorippa indica, a wild crucifer, has been previously reported as the first identified plant in the germplasm of Brassicaceae known to be tolerant towards the mustard aphid Lipaphis erysimi Kaltenbach. We herein report the full-length cloning, expression, purification and characterization of a novel $R$. indica defensin (RiD) and its efficacy against $L$. erysimi. Structural analysis through homology modeling of RiD showed longer a-helix and 3rd $\beta$-sheet as compared to Brassica juncea defensin (BjD). Recombinant RiD and BjD was purified for studying its efficacy against $L$. erysimi. In the artificial diet based insect bioassay, the $L C_{50}$ value of RiD against L. erysimi was found to be $9.099 \pm 0.621 \mu \mathrm{g} / \mathrm{mL}$ which is far lower than that of $B j D(43.51 \pm 0.526 \mu \mathrm{g} / \mathrm{mL})$. This indicates the possibility of RiD having different interacting partner and having better efficacy against L. erysimi over BjD. In the transient localization studies, RiD signal peptide directed the RiD: yellow fluorescent protein (YFP) fusion protein to the apoplastic regions which indicates that it might play a very important role in inhibiting nutrient uptake by aphids which follow mainly extracellular route to pierce through the cells. Hence, the present study has a significant implication for the future pest management program of $B$. juncea through the development of aphid tolerant transgenic plants.
\end{abstract}

Keywords: Aphid tolerance, Brassica juncea defensin, Mustard aphid, Insect pest management, Rorippa indica defensin

\section{Background}

Brassica juncea (Indian mustard) is one of the most important crops cultivated in India. More than three dozen insect pests are found to be associated with mustard crops in India, of which mustard aphid (Lipaphis erysimi Kalt.) is the most dreaded. These pests are able to reduce the yield up to $96 \%$ (Bakhetia and Sekhon 1989; Singh et al. 1982; Dutta et al. 2005a) and oil content by $15-32 \%$ (Kanrar et al. 2002; Verma and Singh 1987) even after good agricultural practices. Besides causing direct feeding injury these aphids vector many plant viruses (e.g., Turnip mosaic virus and Cauliflower mosaic virus) resulting in further yield loss (Moran and Thompson 2001; Moran et al. 2002) which accounts for

\footnotetext{
*Correspondence: samir@jcbose.ac.in

1 Division of Plant Biology, Centenary Campus, Bose Institute,

Kolkata 700054 , India

Full list of author information is available at the end of the article
}

approximately 40,006 million of Indian rupees annually (Kular and Kumar 2011). Moreover, the use of unsustainable chemical pesticides by the farmers has increased which are hazardous to human health as well as to the ecosystem. Earlier studies in developing resistant/tolerant $B$. juncea lines against this particular aphid include production of hybrid lines of B. juncea with Brassica fruticulosa (Atri et al. 2012) and Brassica campestris (Goomber and Labana 1983), selection of vigorous lines from the wild type (Abraham and Bhatia 1994), as well as introducing potent insecticidal agents like proteases (Rahbe et al. 2003) and lectins (Dutta et al. 2005a; Kanrar et al. 2002; Mondal et al. 2006) derived from distant plant families. There is no report of resistant/tolerant gene available in the wild germplasm till date. Moreover, in due course of time, a particular insecticidal agent may become ineffective against the pest due to acquired resistance or behavioural reorientation (Chen 2008), entailing the discovery of a new insecticidal agent. 
Previous study by Bandopadhyay et al. (2013) gave a first molecular insight into crucifer defense response against mustard aphid L. erysimi. On wild germplasm screening, Rorippa indica (L.) Hiern, a wild crucifer and an occasional shade loving weed was noted to be tolerant against mustard aphid. $R$. indica is found in the Indian subcontinent and Asia (Mandal and Sikdar 2003) which remains in rosette form throughout the winter but subsequently bolts out and grows into highly branched bush throughout the summer. Transcriptomic analysis of $R$. indica in response to $L$. erysimi attack was studied by cDNA AFLP analysis in which thirty unique expressed sequence tags (ESTs) were seen to be differentially regulated (Bandopadhyay et al. 2013). One of the major identified ESTs was found to be homologous to PDF1.2c (plant defensin) of Arabidopsis (GenBank Accession-JK034054) which has been named as Rorippa defensin (RiD) for the present study.

Plant defensins are basic, cysteine-rich peptides of about $5-8 \mathrm{kDa}$ (45-54 amino acids) and belong to the $\gamma$-thionin family (Carvalho and Gomes 2009, 2011; Lacerda et al. 2014). Two classes of defensins have been predicted according to the structure and sequences (Lay and Anderson 2005; Lay et al. 2014). Class-I defensin comprises of an endoplasmic reticulum (ER) signal sequence and a mature domain. This class of proteins enter the secretory pathway and do not undergo post-translational modification or subcellular targeting. Class-II defensin contains a C-terminal pro-domain of about 33 amino acids (Lay et al. 2003; Vriens et al. 2014). Many defensins show antifungal (Terras et al. 1992, 1993), antibacterial (Koike et al. 2002; Zhang and Lewis 1997) and insecticidal (Chen et al. 2002) activity. Plant defensins also act as proteinase inhibitors (Melo et al. 2002), protein synthesis inhibitor (Harrison et al. 1997), $\alpha$-amylase inhibitor (Bloch and Richardson 1991), and sodium channel inhibitor (Kushmerick et al. 1998) and can inhibit insect feeding by destroying midgut activity (Carvalho and Gomes 2009; Lin et al. 2007; Pelegrini and Franco 2005; Pelegrini et al. 2008; Santos et al. 2010). Insecticidal and $\alpha$-amylase inhibitory activities of defensins are also reported in Vigna radiata (Liu et al. 2006) as well as in Sorghum bicolor (Bloch and Richardson 1991). Cowpea seed defensin was seen to inhibit $\alpha$-amylase from the weevils-Callosobruchus maculatus and Zabrotes subfasciatus (Santos et al. 2010). BrD1, a defensin gene from Brassica rapa, provides resistance from brown planthopper (Nilaparvata lugens) in transgenic rice (Choi et al. 2009).

In the aforementioned background, the present study was conducted to characterize and study the efficacy of RiD against L. erysimi, over Brassica juncea defensin (BjD). Homology modelling was done to study the differences between the model structures of RiD and $\mathrm{BjD}$. The RiD and BjD coding sequence were cloned, expressed and purified using prokaryotic expression system. For further characterization, transient localization of RiD was studied and finally the insecticidal potential of expressed $\mathrm{RiD}$ as well as that of $\mathrm{BjD}$ was checked through artificial diet based insect bioassay against $L$. erysimi.

\section{Results}

\section{Aphid infestation study}

Time course aphid population study was carried out for up to 7 days. After infesting each plant with 30 aphids, a settling time of $2 \mathrm{~h}$ was allowed. Following this, the number of viable wingless aphids per plant was counted at each day post infestation. A surge in the population of nymphs and aptera was noted within 4 days post infestation (dpi) (Fig. 1). After 4 dpi, population in $R$. indica started to decline sharply with the appearance of the winged (alate) form of the aphids. Among all the sets of infested and non-infested plants as shown in Fig. 1, $R$. indica was seen to have lower aphid population with increasing days post infestation than $B$. juncea. The challenged $R$. indica plants were found to show normal development like the non-infested control plants (Additional file 1: Fig. S1).

\section{Time course semi-quantitative RT-PCR and real-time PCR analysis}

Semi-quantitative expression of PDF1.2c homolog in $R$. indica as well as in $B$. juncea was studied using the primers from the EST as previously described (Additional file 4: Table S1). The RT-PCR profile is presented in Fig. 2a. A 1.5-fold up-regulation was observed in $R$. indica early at $6 \mathrm{~h}$ as compared to 1.2 -folds in $B$. juncea and the expression culminated to 48.2-folds in $R$. indica

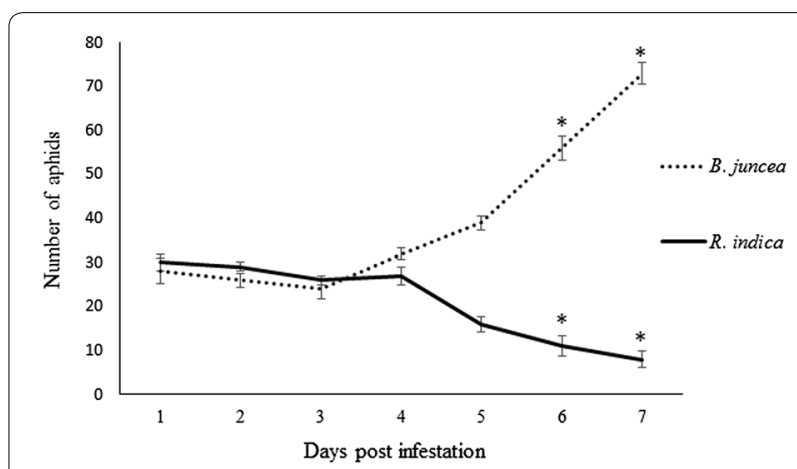

Fig. 1 Aphid population on R. indica and B. juncea. The total number of live aphids were recorded over 7 days post infestation at an interval of $24 \mathrm{~h}$. Maximum colonization was observed in case of $B$. juncea. Whereas a decrease in population of aphids was seen in $R$. indica after $4 \mathrm{dpi}$. Bars represent standard error (SE) where number of independent experiments $(n)=3$. The significant changes $(P \leq 0.05)$, marked by asterisk were analyzed by Student's t test 

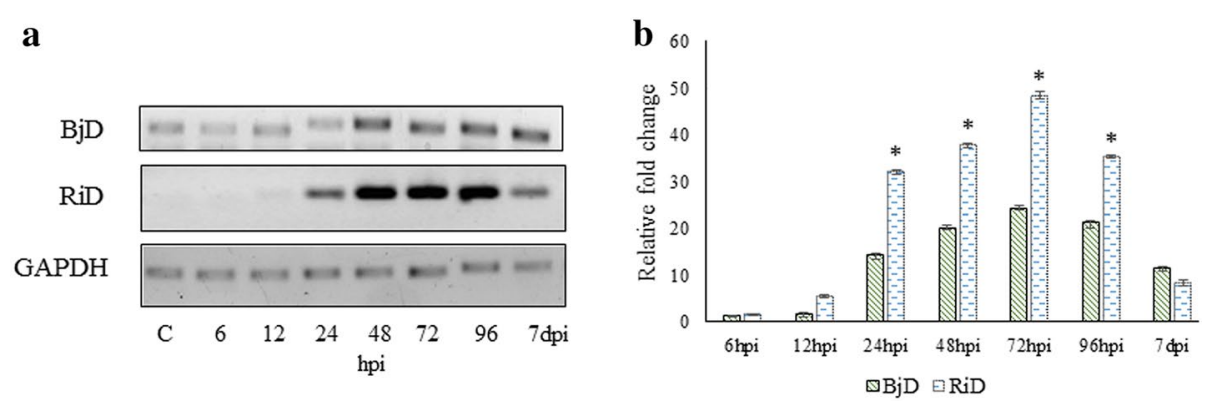

Fig. 2 a Semi quantitative PCR analysis of PDF1.2c homolog in R. indica (RiD) and B. juncea (BjD) at different time points. b Real time expression profile of PDF1.2c homolog in R. indica and B. juncea upon aphid infestation. Bars represent standard error (SE) of three biological replicates (n). The significant changes $(P \leq 0.01)$ were marked by asterisk (analyzed by Student's $t$ test)

by $72 \mathrm{~h}$ post infection (hpi) through 9.45 and 9.67 -fold at 24 and 48 h respectively (Fig. 2b). Whereas, PDF1.2c homolog expression in $B$. juncea reached a peak not more than 24.25 -folds by $72 \mathrm{hpi}$ and declined to 11.5 -folds by 7 dpi. The expression level of PDF1.2c homolog in $R$. indica drops to 8.2 -folds by $7 \mathrm{dpi}$ (Fig. 2b).

\section{Determination of the full-length coding sequence of RiD and $B j D$ and in silico analysis}

$5^{\prime}$ and $3^{\prime}$ RACE was carried out to identify the full-length coding sequence of $R$. indica defensin (243 bp) and $B$. juncea defensin (243 bp). A single intron in the RiD gene and a short region of $393 \mathrm{bp}$ upstream element containing TATA box and other cis-acting elements were also identified (Fig. 3) using the protocols of genome walk. The coding sequences and upstream elements of RiD and
BjD were submitted to GenBank with accession numbers KP893333 and KU513489 respectively.

The sequence of RiD was aligned with other plant defensins of the same family-Brassicaceae along with BjD. RiD showed $87,88,85,86$ and $86 \%$ identity with the defensins from B. juncea, Raphanus sativus (GenBank accession-U18557), Arabidopsis (GenBank accession-NM106233.3), Sinapis alba (GenBank accession-AY998243) and Brassica rapa (GenBank accessionXM009106448) respectively at nucleotide level (Fig. 4).

\section{Homology modelling and structure determination}

$\mathrm{RiD}$ and $\mathrm{BjD}$ both showed $98 \%$ sequence identity at amino acid level with $R$. sativus antifungal protein 1 (PDB ID: 1AYJ). Homology modelling clearly shows that despite having $86 \%$ similarity at the amino acid level, the

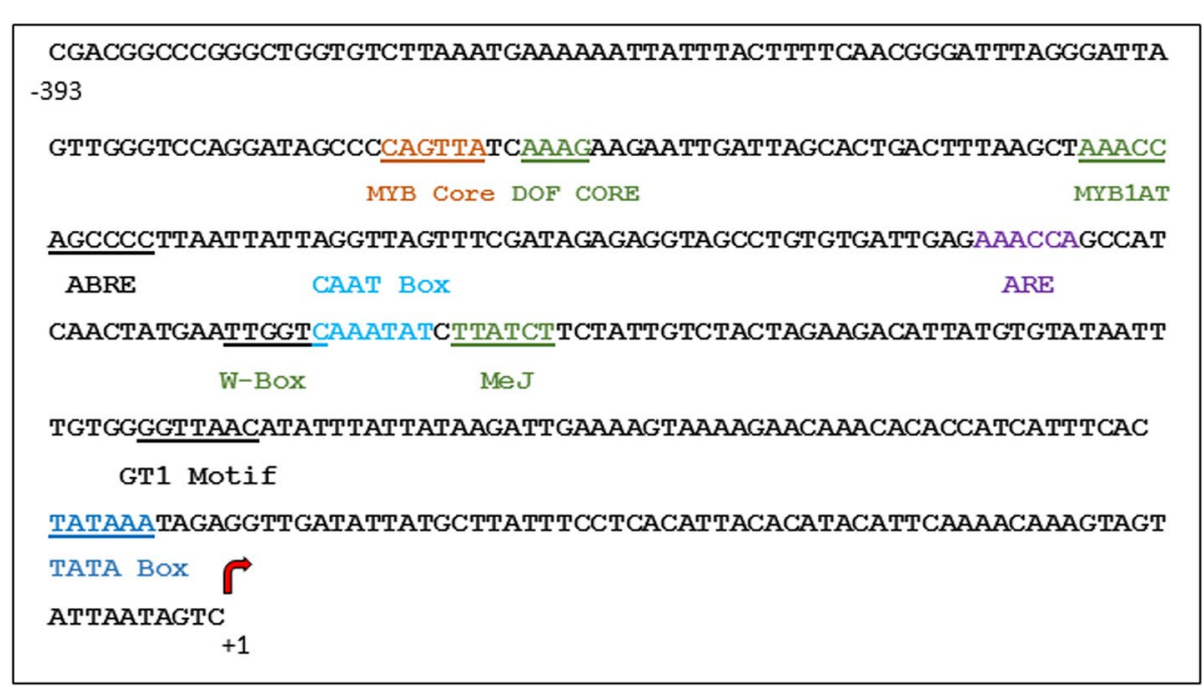

Fig. 3 In silico promoter analysis showing important cis acting elements. A 393 bp region was analyzed for upstream elements (ARE: cis-acting regulatory element essential for the anaerobic induction, ABRE: abscisic acid response element, DOF Core: DNA-binding domain with one finger transcription factors, GT1 Motif: light responsive element, I-Box: part of a light responsive element, MeJ: methyl jasmonate inducing region, MYB Core: MYB transcription factor binding region, MYB1AT: dehydration responsive element, TATA Box: core promoter element around -30 of transcription start, W-Box: binding sites for WRKY transcription factors) 


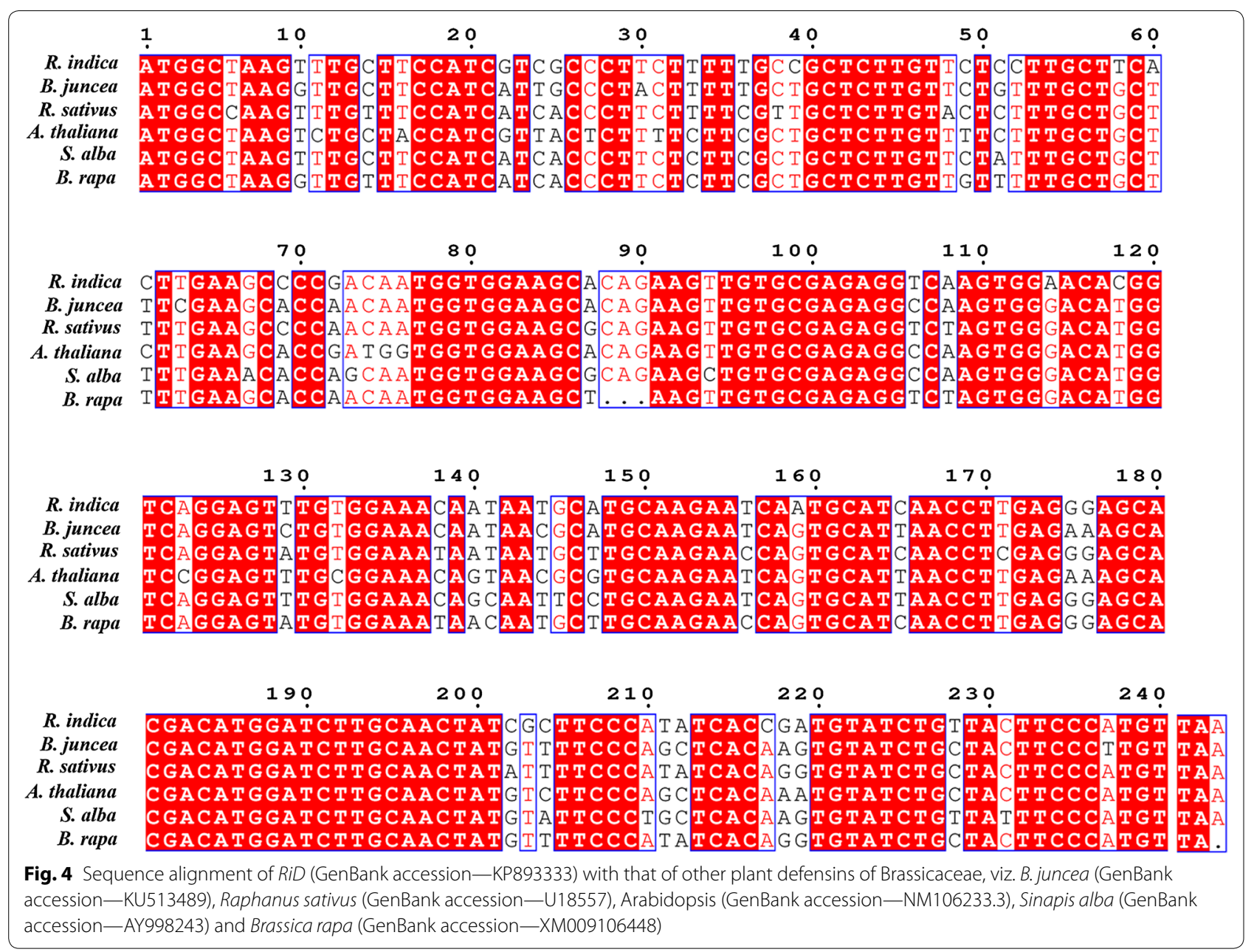

$\alpha$-helix and 3rd $\beta$-sheet of RiD are little longer as compared with $\mathrm{BjD}$ (Fig. $5 \mathrm{a}-\mathrm{c}$ ).

\section{Adaptive Poisson-Boltzmann Solver (APBS) iso-surface calculations}

The electrostatic potential and distribution of atomic partial charge of biomacromolecule in connection with dielectric constant could be explained by APBS. The Adaptive Poisson-Boltzmann Solver (APBS) was used to calculate the electrostatic surface of the proteins. For both $\mathrm{RiD}$ and $\mathrm{BjD}$, the outer surfaces of the proteins were seen to have more positive charge (Fig. $5 \mathrm{~d}-\mathrm{g}$ ).

\section{Protein expression and purification}

The recombinant $\mathrm{pET} 28 \mathrm{a}+$ vectors harbouring the coding sequence of $R i D$ and $B j D$ were transformed into Rosetta (DE3) pLysS cells individually. The expression constructs produced a $6 \mathrm{His}-\mathrm{RiD}$ and $6 \mathrm{His}-\mathrm{BjD}$ fusion protein respectively, which were purified by $\mathrm{Ni}-\mathrm{NTA}$ column. The maximum expression of $\mathrm{RiD}$ and $\mathrm{BjD}$ was obtained at $1 \mathrm{mM}$ IPTG at $20^{\circ} \mathrm{C}$ after $16 \mathrm{~h}$ of incubation. As shown in Fig. 6a, lane 2 and lane 3, bands of approximately $9 \mathrm{kDa}$ of both $\mathrm{RiD}$ and $\mathrm{BjD}$ were eluted from the induced clones in $12 \%$ SDS-PAGE gel. The purified proteins were further validated by western blot analysis using anti-His antibody (Fig. 6a, lanes 4 and 5).

\section{Mass spectrometric analysis of purified RiD}

A MALDI-TOF/TOF-MS analysis was performed to verify the molecular mass of the purified RiD protein. MS analysis yielded a mass of 9067.364 Da for the purified peptide (Fig. 6b). The molecular mass of $\mathrm{RiD}$ is $\sim 6 \mathrm{kDa}$ and the size of the tag (6His) associated with the defensin is $\sim 3 \mathrm{kDa}$. Thus, the recombinant protein obtained was of $\sim 9 \mathrm{kDa}$. The MALDI-TOF/TOF-MS spectrum was generated from in-solution trypsin digestion (Additional file 2: Fig. S2). Furthermore, analyses by the MASCOT search program in the NCBI database suggested that the obtained sequence is highly similar with $R$. indica defensin, and other defensin-like proteins. The observed 
$\mathbf{a}$

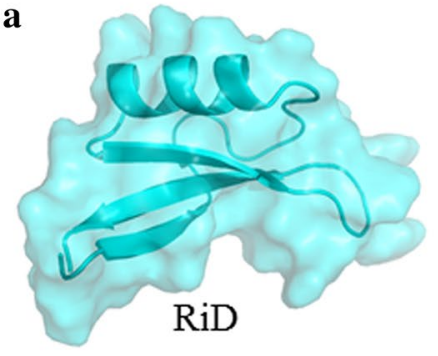

d
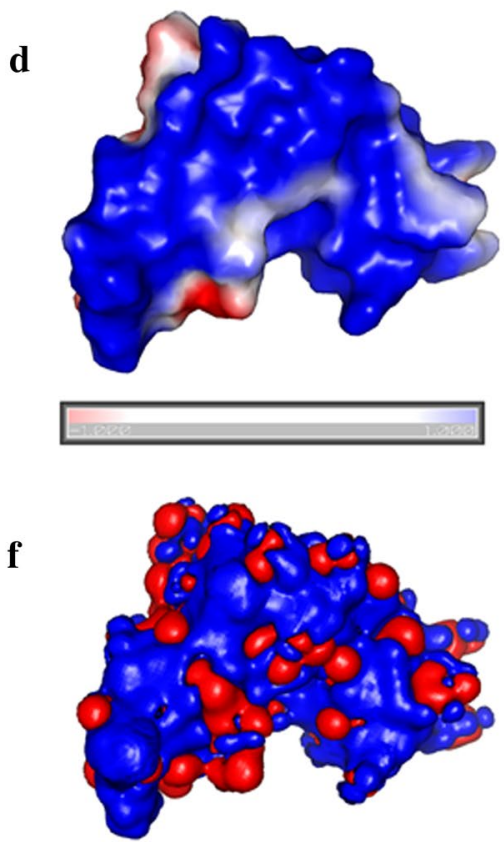
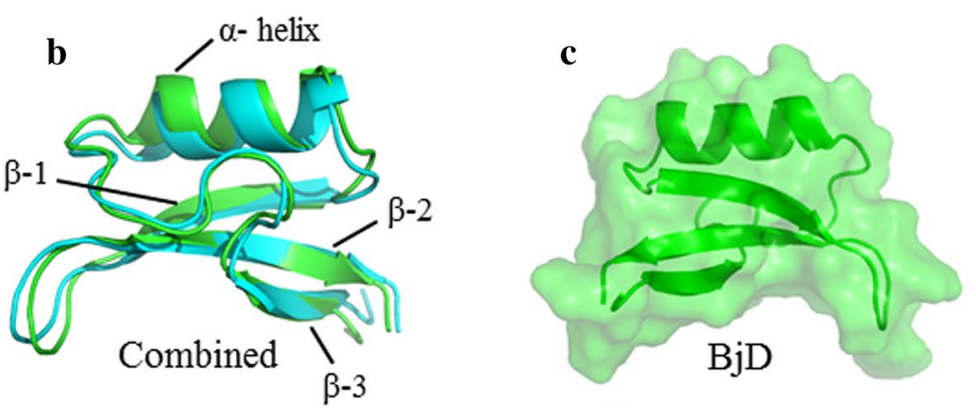

e

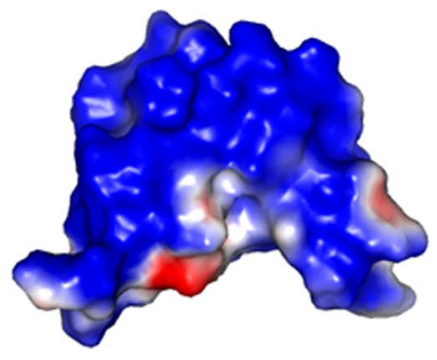

g

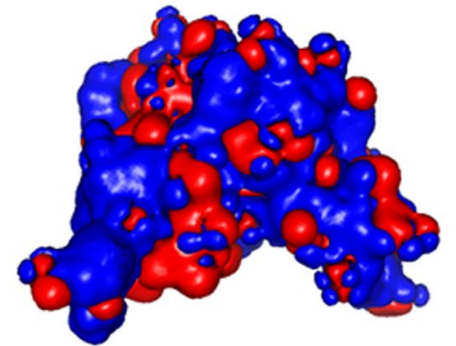

Fig. 5 Homology model structure of $\operatorname{RiD}(\mathbf{a})$ and $\operatorname{BjD}(\mathbf{c})$ showing one $a$-helix and three $\beta$-sheet region. Superimposed homology model structure of RiD (cyan) and BjD (green) (b). Color by potential of solvent accessible surface of RiD (d) and BjD (e) have been calculated using APBS. The bars indicates color by potential [positive (b/ue) and negative (red)]. Electrostatic iso-surfaces of RiD (f) and BiD (g) are also shown

monoisotopic mass of tryptically digested peptides obtained by MALDI-TOF/TOF-MS and their position with respect to matched protein sequence are presented in Table 1 . The matched peptides of RiD covered $33 \%$ of defensin ( $R$. indica, gi694199016) with score 47 and $48 \%$ with defensin-like protein 1 (Camelina sativa, gi727556613) with score 50.

\section{Localization of RiD}

All class-I defensin have a predicted N-terminal signal peptide supposed to translocate the defensin into the lumen of the endoplasmic reticulum (ER) and thus to the secretory pathway. A SignalP analysis (Petersen et al. 2011) of the $R$. indica defensin showed that RiD also has a putative $\mathrm{N}$-terminal secretory signal peptide (Additional file 3: Fig. S3a). In order to determine the function of this putative defensin, chimeric proteins were constructed with yellow fluorescent protein (YFP) fused to the C-terminus of the full-length RiD (RiD: YFP) (Additional file 3: Fig. S3b) and only YFP lacking RiD was used as a control. RiD: YFP labelling was observed in the apoplastic regions of the onion epidermal while fluorescence from control YFP was seen to be diffused in the cytoplasm (Fig. 7).

\section{Insect bioassay on artificial diet}

Mortality of $L$. erysimi was studied using artificial liquid diet supplemented with different concentrations of RiD (Fig. 8a) and BjD (Fig. 8b). Liquid diet with no RiD and no BjD was used as control. The total mortality $\left(P^{\prime}\right)$ was calculated from the Abbot's formula (Abbott 1925), $P^{\prime}=C+P(1-C)$, considering a population $(P)$ which would have survived in the absence of the insecticidal agent used in the experiment and where $C$ is natural mortality without the agent. $\mathrm{LC}_{50}$ value of $\mathrm{RiD}$ and $\mathrm{BjD}$ against $L$. erysimi were calculated accordingly using the 


\section{a}

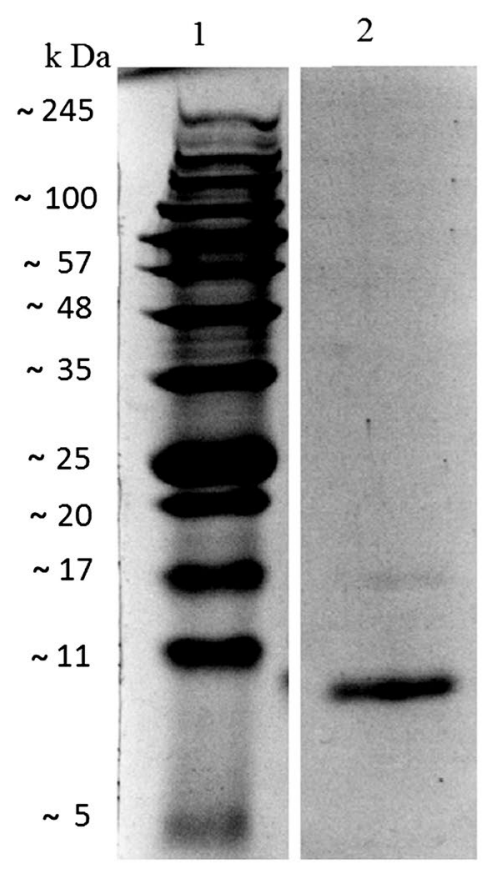

3

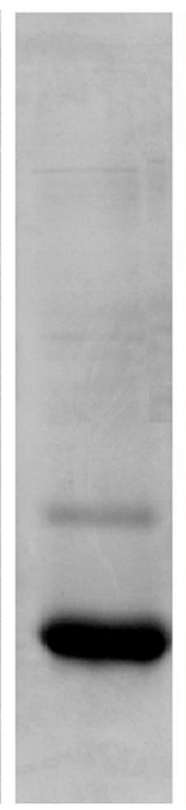

5

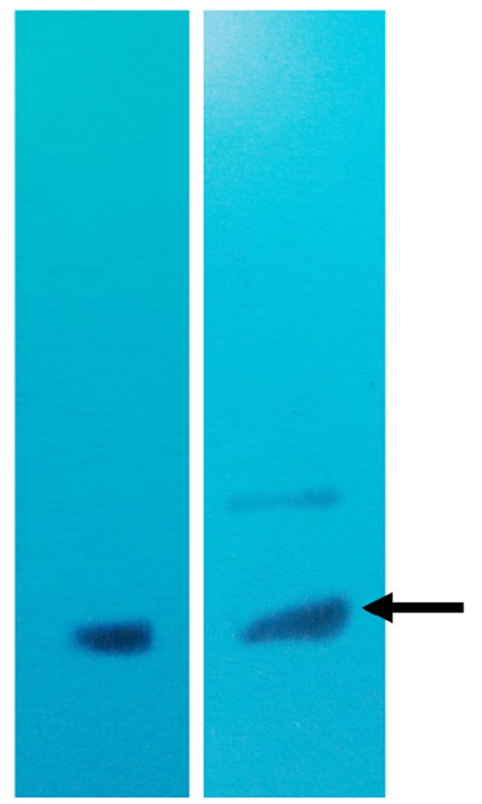

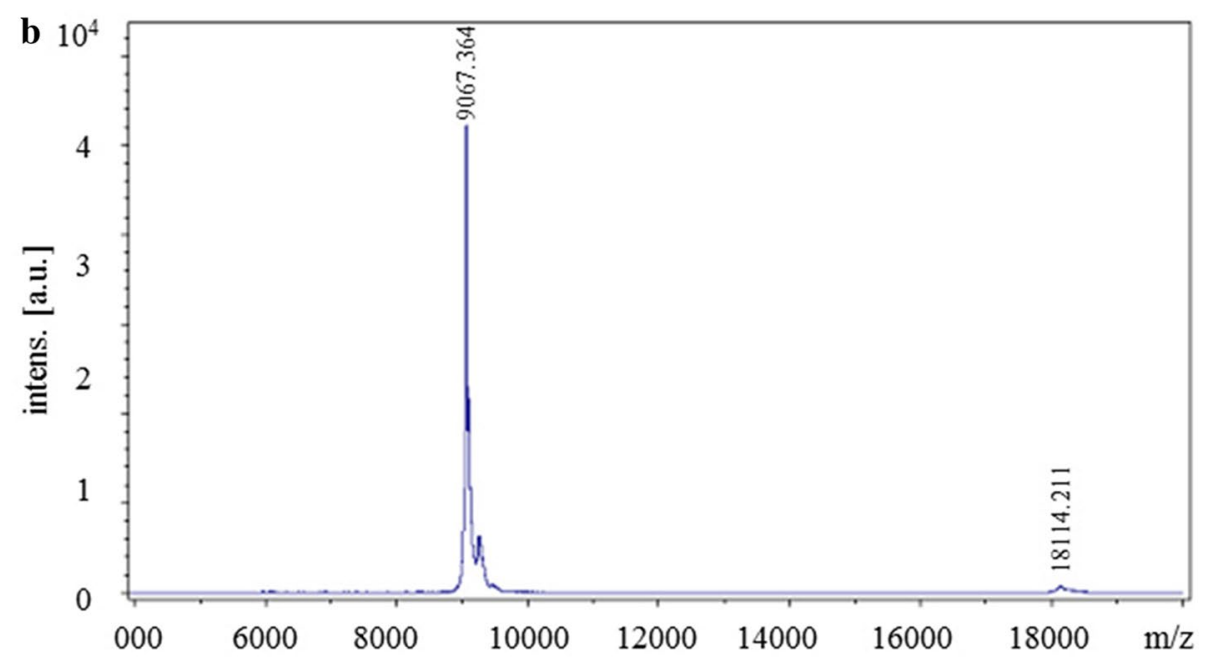

Fig. 6 Purification of RiD and BjD. a Lane 1 Protein marker, Lane 2 purified RiD, Lane 3 purified BjD, Lane 4 Western blot using anti-His antibody (1:5000) against purified RiD, Lane 5 Western blot using anti-His antibody (1:5000) against purified BjD. Arrow indicates purified proteins. b Determination of RiD molecular mass by MALDI-TOF/TOF-MS spectrometry. A sharp peak confirms the quality of purification of RiD

statistical Probit analysis. $\mathrm{LC}_{50}$ value of RiD against $L$. erysimi was found to be $9.099 \pm 0.621 \mu \mathrm{g} / \mathrm{mL}$, whereas of $\mathrm{BjD}$ was found to be $43.51 \pm 0.526 \mu \mathrm{g} / \mathrm{mL}$ (Table 2).

\section{Discussion}

Plant defensins are known to have a diverse range of functions. They are one of the most important components of the innate immune system that have been conserved during evolution. Among the limited insect resistance genes like Bacillus thuringiensis (Bt) genes (Lawo et al. 2009; Porcar et al. 2009), proteases (Rahbe and Febvay 1993) and lectins (Bandopadhyay et al. 2001; Kanrar et al. 2002; Banerjee et al. 2004; Dutta et al. 2005a, b; Mondal et al. 2006; Das et al. 2013), till date only lectins have been studied to be effective against L. erysimi (Dutta et al. 2005b; Kanrar et al. 2002; Mondal et al. 2006; Roy et al. 2014). Defensins with $\alpha$-amylase inhibitory activity are potential candidates which can possibly 
Table 1 Summary of matched peptides of RiD analyzed by MALDI-TOF/TOF-MS

\begin{tabular}{|c|c|c|c|c|c|}
\hline Protein name/accession number & Theoretical pl & Score & Peptide mass (Da) & Position & Matched peptides \\
\hline \multirow[t]{4}{*}{ Defensin (Rorippa indica, 694199016) } & \multirow[t]{4}{*}{8.71} & \multirow[t]{4}{*}{47} & 507.3724 & $36-40$ & R. SSGTR.S \\
\hline & & & 719.4432 & $69-73$ & R. FPYHR. C \\
\hline & & & 1174.6655 & $52-61$ & K. NQCINLEGAR.H \\
\hline & & & 1593.8500 & $62-73$ & R.HGSCNYRFPYHR.C \\
\hline \multirow[t]{4}{*}{ Defensin-like protein 1 (Camelina sativa, 727556613) } & \multirow[t]{4}{*}{8.42} & \multirow[t]{4}{*}{50} & 719.4432 & $67-71$ & R.FPYHR.C \\
\hline & & & 1174.6655 & $50-59$ & K.NQCINLEGAR.H \\
\hline & & & 1593.8500 & $60-71$ & R.HGSCNYRFPYHR.C \\
\hline & & & 1698.8252 & $34-49$ & R.SSGTWSGVCGNNNACK.N \\
\hline \multirow[t]{4}{*}{ Defensin-like protein 3 (Camelina sativa, 727556613) } & \multirow[t]{4}{*}{8.42} & \multirow[t]{4}{*}{49} & 719.4432 & $68-72$ & R.FPYHR.C \\
\hline & & & 1174.6655 & $51-60$ & K.NQCINLEGAR.H \\
\hline & & & 1593.8500 & $61-72$ & R.HGSCNYRFPYHR.C \\
\hline & & & 1698.8252 & $35-50$ & R. SSGTWSGVCGNNNACK.N \\
\hline
\end{tabular}

\section{RiD: YFP}
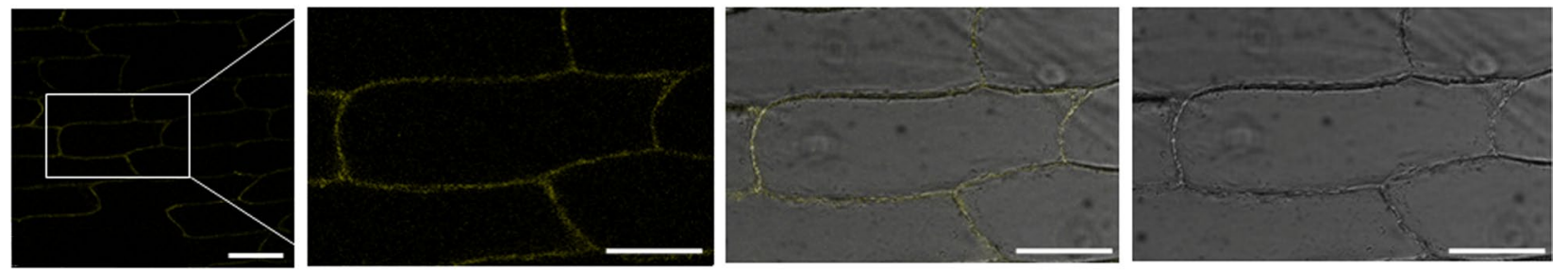

YFP (control)
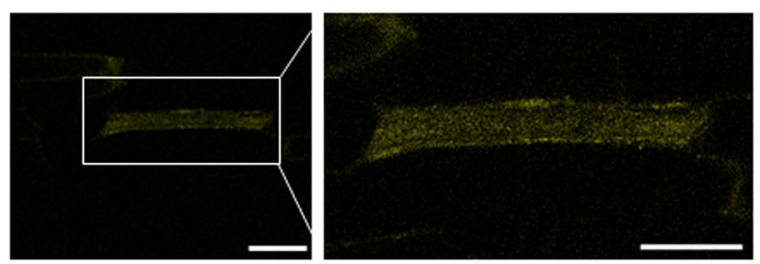

b

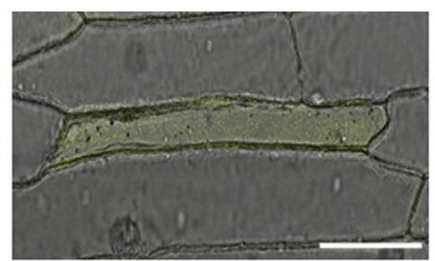

c

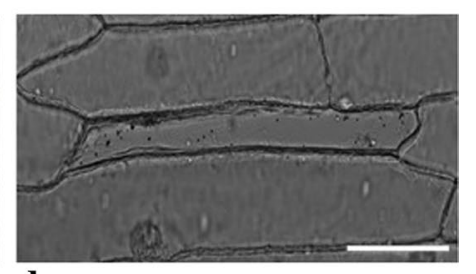

d

Fig. 7 Localization studies of RiD:YFP and YFP only (as control) in onion epidermal cells. Confocal laser sections show YFP fluorescence in onion epidermal cells expressing RiD:YFP in the apoplastic regions and diffused fluorescence in the cells expressing only YFP. a Fluorescence, $\mathbf{b}$ magnified fluorescence, $\mathbf{c}$ merged, $\mathbf{d}$ bright field. (Magnification: $\times 20$ ). Bar $75 \mu \mathrm{m}$

provide resistance against insect feeding (Pelegrini et al. 2008) and can be a promising tool in transgenic development strategies in this present scenario.

In the present study, a novel defensin gene-RiD has been isolated and characterized from $R$. indica, previously noted to be aphid tolerant by Bandopadhyay et al. (2013). RiD is seen to be highly upregulated when infested upon by the mustard aphid-L. erysimi (Bandopadhyay et al. 2013). Here, RiD and defensin from $B$. juncea (BjD) has been successfully cloned, expressed, purified and characterized. Analysis of $\mathrm{RiD}$ and $\mathrm{BjD}$ through homology modelling showed structural difference through the presence of longer $\alpha$-helix and 3rd $\beta$-sheet in the former one, despite having significant similarity in nucleotide level. Plant defensins are reported to have a broad spectrum of biological activities (Lacerda et al. 2014). Reports of VrD1 from Vigna radiata is shown to inhibit insect $\alpha$-amylase whereas $\operatorname{VrD} 2$ from the same plant does not have any insecticidal activity nor $\alpha$-amylase inhibitory activity (Lin et al. 2007). There is also a family of defensins isolated from Vigna unguiculata, in which different homologous forms may act as antifungal, antibacterial, and enzyme inhibitors (Franco 2011). Thus, $\mathrm{RiD}$ being very identical to $\mathrm{BjD}$ might have a different mode of action and efficacy against $L$. erysimi.

Transient expression of RiD fused with YFP showed that $\mathrm{RiD}$ is secreted into the apoplast and not retained in the intracellular compartments. Secretion into the 

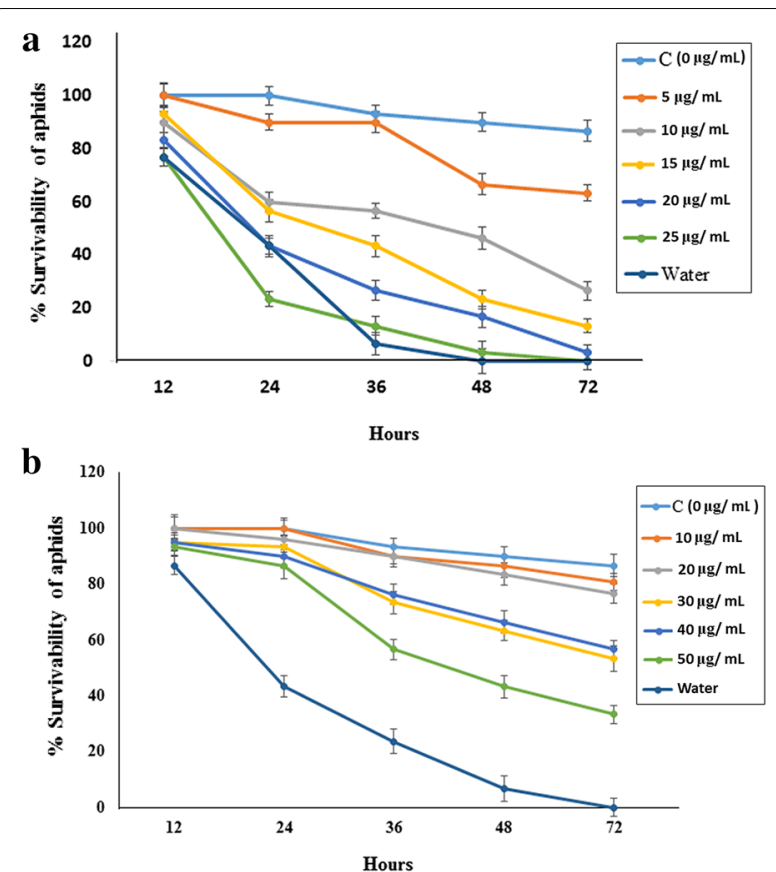

Fig. 8 Insect bioassay in artificial diet supplemented with RiD and BjD on second instar nymphs of $L$. erysimi. Insect survivability graph at different concentrations of $\mathbf{a} \operatorname{RiD}(0,5,10,15,20,25 \mu \mathrm{g} / \mathrm{mL})$ and $\mathbf{b} \mathrm{BjD}$ $(0,10,20,30,40,50 \mu \mathrm{g} / \mathrm{mL})$ were recorded over $72 \mathrm{~h}$

apoplast is consistent with the expected function of the signal peptide of all Class-I defensins, allowing a posttranslational translocation into the lumen of ER. This is in accordance with other plant defensins (Kaur et al. 2012; Kragh et al. 1995) and contradictory to AhPDF1.1 of Arabidopsis, where it is retained in the vacuoles (Oomen et al. 2011). As aphid stylets follow mainly extracellular pathway through the apoplast to obtain nutrition (Jaouannet et al. 2014; Will and Vilcinskas 2015) RiD might play a very important role in inhibiting sap sucking by aphids.

To determine the insecticidal efficacy of RiD against $L$. erysimi, it is essential to calculate the $\mathrm{LC}_{50}$ value of the protein against the target insect. Hence, artificial diet based bioassay was conducted and it was observed that $\mathrm{RiD}$ induced mortality in L. erysimi with $\mathrm{LC}_{50}$ value of $9.099 \pm 0.621 \mu \mathrm{g} / \mathrm{mL}$. Similar bioassay conducted using $\mathrm{BjD}$ yielded $\mathrm{LC}_{50}$ value of $43.51 \pm 0.526 \mu \mathrm{g} / \mathrm{mL}$, indicating that $\mathrm{BjD}$ is not at all effective against this aphid. The
$\mathrm{LC}_{50}$ value of $\mathrm{RiD}$ was also observed to be lower than that of other insecticidal agents such as Amorphophallus paeonifolius tuber agglutinin (AMTL) with $\mathrm{LC}_{50}$ value of $13.47 \pm 0.23 \mu \mathrm{g} / \mathrm{mL}$ (Mondal et al. 2012), Allium sativum leaf agglutinin (ASAL) with $\mathrm{LC}_{50}$ value $20.7 \pm 0.21 \mu \mathrm{g} /$ $\mathrm{mL}$ (Banerjee et al. 2011), Colocasia esculenta tuber agglutinin (CEA) with $\mathrm{LC}_{50}$ value of $11.87 \pm 0.229 \mu \mathrm{g} / \mathrm{mL}$ (Roy et al. 2014) and proteases with $\mathrm{LC}_{50}$ value of $22 \mu \mathrm{g} /$ $\mathrm{mL}$ (Harrison and Bonning 2010). This indicates the suitability of RiD as an insecticidal agent over $\mathrm{BjD}$ and other previously reported agents against L. erysimi. Further studies in identifying any interacting partners from $L$. erysimi might provide a better insight into the mechanism of RiD. To the best of our knowledge, this is the first report of characterization of a putative mustard aphid tolerant gene from the Brassicaceae germplasm.

\section{Conclusion}

In the present study, $\mathrm{RiD}$ and $\mathrm{BjD}$ were isolated from $R$. indica and B. juncea respectively. Despite the high level of homology on sequence level with $\mathrm{BjD}, \mathrm{RiD}$ has been seen to differ in its structural and surface properties as well as its efficacy against L. erysimi, suggesting that $\mathrm{RiD}$ could be a potent insecticidal agent. Hence, the present study might be promising for the development of aphid tolerance in $B$. juncea through the generation of transgenics.

\section{Methods}

\section{Experimental set up}

Rorippa indica and B. juncea plants of 60 days old were grown under laboratory conditions under $16 \mathrm{~h}$ photoperiodic light (3000 lux), temperature of $25 \pm 2{ }^{\circ} \mathrm{C}$ and $75 \%$ relative humidity. Mustard aphids (L. erysimi) were collected from the Madhyamgram Experimental Farm, Bose Institute, India. Thirty aphids of third-instar stage were forcedly infested upon each plant (under laboratory conditions previously mentioned), which were used for further experiments. Three plants with 30 aphids each were used as replicates.

\section{Aphid population}

The total aphid population was studied in both $R$. indica and $B$. juncea at an interval of every $24 \mathrm{~h}$ for 7 days, with three plants for each time point. Student's t test at a significance level of $\mathrm{P} \leq 0.05$ was carried out to determine the significance of the infestation noted over the time course.

Table 2 LC $_{50}$ value of purified RiD and BjD against Lipaphis erysimi in artificial diet based bioassay

\begin{tabular}{llllllll}
\hline Protein used & $\mathbf{L C}_{\mathbf{5 0}}$ value $(\boldsymbol{\mu g} / \mathbf{m L})$ & Lower fiducial limit & Upper fiducial limit & SE of slope & Regression equation $\mathbf{Y}$ & $\mathbf{X}^{\mathbf{2}}$ value & $\mathbf{d f}$ \\
\hline RiD & 9.099 & 13.726 & 3.274 & 0.621 & $7.921+(-3.046) \times$ & 3.816 & 3 \\
BjD & 43.51 & 48.321 & 37.624 & 0.526 & $1.77+1.60 \times$ & 1.210 & 3 \\
\hline
\end{tabular}




\section{RNA isolation, cDNA preparation and rapid amplification of cDNA ends}

Total RNA was isolated from the aerial parts (leaves and stem) of all the infested plants at different time points as well as the non-infested control plants using Trizol reagent (Invitrogen, CA, USA). First strand cDNA was synthesized using iScript-cDNA synthesis kit (Bio-Rad, CA, USA). The obtained cDNA was used for semi-quantitative analysis for the expression of PDF1.2c homolog and realtime PCR analysis using iQ SYBR Green Supermix (BioRad Laboratories) in a iQ5 Multicolor Real-Time PCR Detection System (Bio-Rad Laboratories). Primers were designed on the basis of the previously identified EST (GenBank Accession-JK034054) (Bandopadhyay et al. 2013). The reactions were studied at all seven time-points with three different biological replicates. The real-time RT-PCR condition used was as follows: initial denaturation at $95^{\circ} \mathrm{C}(3 \mathrm{~min})$, followed by 50 cycles $(10 \mathrm{~s})$ at $95^{\circ} \mathrm{C}$ and $30 \mathrm{~s}$ at $55^{\circ} \mathrm{C}$. GAPDH was used as an internal control. The average fold value changes for the different time points were calculated using $2^{-\Delta \Delta C t}$ method (Livak and Schmittgen 2001). The full-length mRNA sequence was determined using $5^{\prime}$ and $3^{\prime}$ RACE System for rapid amplification of cDNA ends (Invitrogen, CA, USA).

\section{Genomic DNA isolation and genome walk}

Genomic DNA of $R$. indica was isolated using DNeasy Plant maxi kit (Qiagen, Hilden, Germany). Genome walk was carried out according to the protocols of BD genome Walker $^{\mathrm{TM}}$ Universal Kit (BD Biosciences Clontech, CA, USA). The primers were designed according to the sequence derived from RACE (Additional file 4: Table S1). PCR product was purified using Qiaquick Gel Extraction kit (Qiagen, Hilden, Germany) and was cloned into pGEMT Easy Vector (Promega, Wisconsin, USA). Nucleotide sequencing of the clones was performed using Applied Biosystems (USA) $3130 x l$ Genetic Analyzer at the sequencing facility of Bose Institute, Kolkata, India. RiDForward and RiDReverse primers were used to clone the full-length gene sequence of RiD (Additional file 4: Table S1).

\section{In silico analysis}

The coding sequences of $R i D$ and $B j D$ derived from RACE were analyzed in TAIR and NCBI resources using BLASTN algorithm (Altschul et al. 1990). All the sequences derived from the Brassicaceae family were aligned using ClustalW (Thompson et al. 1994) and ESPript 3.0 (Robert and Gouet 2014). The intron in RiD gene was identified using GENSCAN (Burge and Karlin 1997) as well as aligning the sequence to the full-length cDNA sequence. The sequence was also analyzed using PLACE (Higo et al. 1998) and PlantCARE (Lescot et al. 2002) for identification of upstream elements.

\section{Homology modelling}

The full-length coding sequence of $R i D$ and $B j D$ were translated using ExPASy translate tool. The primary amino acid sequences thus derived were taken to investigate for a proper template in Protein Data Bank (PDB) to generate $3 \mathrm{D}$ coordinates of both $\mathrm{RiD}$ and $\mathrm{BjD}$. Both the sequences were searched for a structure using Prime v3.4 module of Schrödinger molecular modelling suite (Jacobson et al. 2004). BLAST homology search was performed against non-redundant protein databank. The structures derived from homology modelling were further energy minimized using Polak-Ribiere conjugate gradient (PRCG) method, where the maximum iteration steps were 2500 . The whole method was performed using OPLS_2005 force field (Jorgensen et al. 1996). The structure derived for $\mathrm{RiD}$ was also aligned with that of $\mathrm{BjD}$ in PyMol to identify the regions of dissimilarity present if any.

\section{Adaptive Poisson-Boltzmann Solver (APBS) iso-surface calculations}

The electrostatic characteristics of the protein were analyzed using APBS (Baker et al. 2001). The AMBER force field was used for the generation of standard all-atom charges. The various computations necessary to calculate the electrostatic properties were performed using a plugin option of PyMOLv1.2r3pre. The electrostatic contour visualizations were collected at the suitable positive and negative iso-surface values, optimized for visualization.

\section{Protein expression and purification}

The full-length coding sequence of $R i D$ and $B j D$ encoding the mature peptide was cloned into the pET28a+ vector individually (Novagen, WI, USA) using RiDexpF/RiDexp-R (for RiD) and BjDexp-F/BjDexp-R (for BjD) containing the $B a m \mathrm{HI}$ (in forward primers, underlined) and $\mathrm{SacI}$ (in reverse primers, underlined) sites (Additional file 4: Table S1). The recombinant plasmids were then transformed into the E. coli Rosetta (DE3) pLysS cell line (Novagen, WI, USA). The recombinant cells were induced with $1 \mathrm{mM}$ isopropyl- $\beta$-D-thiogalactopyranoside (IPTG) and incubated with constant shaking at $20{ }^{\circ} \mathrm{C}$ for $16 \mathrm{~h}$. The recombinant cells were pelleted by centrifugation at $5000 \mathrm{~g}$ at $4{ }^{\circ} \mathrm{C}$ for $30 \mathrm{~min}$, resuspended in lysis buffer $\left(50 \mathrm{mM} \mathrm{NaH} \mathrm{PO}_{4}, 300 \mathrm{mM} \mathrm{NaCl}, 10 \mathrm{mM}\right.$ imidazole, $\mathrm{pH}$ 7.4) and sonicated 30 times for $30 \mathrm{~s}$ each. The cell suspension was centrifuged at $10,000 \mathrm{~g}$ for $30 \mathrm{~min}$ at $4{ }^{\circ} \mathrm{C}$, and the supernatant was incubated for $2 \mathrm{~h}$ in $2 \mathrm{~mL}$ of nickel-nitrilotriacetic acid (Ni-NTA) column equilibrated with lysis buffer. The column was washed with wash buffer with $20 \mathrm{mM}$ imidazole, $\mathrm{pH} 7.4$ to remove nonspecific proteins, and finally, the target fusion protein 
was eluted with elution buffer with $250 \mathrm{mM}$ imidazole, $\mathrm{pH}$ 7.4. All purification steps were carried out at $4{ }^{\circ} \mathrm{C}$. The expression and purification of the recombinant proteins were analyzed in $12 \%$ SDS-PAGE stained with Coomassie Brilliant Blue. The expressions of the proteins were further confirmed by western blot analysis using anti-His antibody (1:5000) (Biobharati, Kolkata, India).

\section{MALDI-TOF/TOF-mass spectrometric analysis}

Protein structures may lead to anomalously fast migration in SDS-PAGE (Jong et al. 1978, Rath et al. 2009). To determine the exact molecular mass, purified RiD was analyzed on a matrix-assisted laser desorption/ionization time-of-flight Autoflex III mass spectrometer (Bruker Daltonics, Germany). Samples were prepared by mixing equal volumes of $0.1 \%$ trifluoroacetic acid (TFA), acetonitrile (1:1) and the protein solution. A $2 \mu \mathrm{L}$ portion of the above sample was mixed with $2 \mu \mathrm{L}$ of freshly prepared $\alpha$-cyano-4-hydroxycinnamic acid (HCCA) matrix in $50 \%$ acetonitrile and $1 \%$ TFA (1:1), and $2 \mu \mathrm{L}$ was spotted on the target Anchor Chip MALDI plate (Bruker Daltonics, Germany). The protein was also digested with trypsin (Promega, Madison, USA) according to the in solution digestion protocol of Mann (2006). The spectra obtained were analyzed with Flex Analysis Software (version 2.4, Bruker Daltonics, Germany) for deletion of matrix peaks and tryptic autolysis peaks. Processed spectra were then searched using MS Biotools (version 3.2) program against the taxonomy Viridiplantae (Green plants).

\section{In situ localization}

The full-length coding sequence of RiD was cloned into pENTR D-TOPO vector (Invitrogen, CA, USA) using RiDloc-F and RiDloc-R (Additional file 4: Table S1) according to the manufacturer's instructions. And then it was cloned into destination vector pEARLY GATE 101 (CD3-683, YFP) using LR clonase (Invitrogen, CA, USA). YFP was chosen over GFP due to its higher photo-stability and improved brightness (Shaner et al. 2005). The destination clone was finally coated with gold particles (Bio-Rad, CA, USA) and was bombarded into onion epithelial cells using PDS-1000/He System (Bio-Rad, CA, USA). The cells were observed after $24 \mathrm{~h}$ in Leica Zeiss Confocal Laser scanning microscope at $488 \mathrm{~nm}$ excitation and fluorescence emission signal recovery between 505 and $535 \mathrm{~nm}$.

\section{Artificial diet based bioassay of $L$. erysimi with purified RiD and BjD}

Second instar nymphs were used for this experiment. Artificial diet was formulated using the original description by Dadd and Mittler (1976). The experiment was set up in small $35 \mathrm{~mm}$ petri dishes (Tarsons, Kolkata,
India) with small perforated bottom just to allow the passage of air. Twenty nymphs of $L$. erysimi were incubated for each set and the petri dishes were covered with parafilm stretched 4 times to its original size. A liquid diet of $400 \mu \mathrm{L}$ supplemented with different doses of $\mathrm{RiD}$ $(0,5,10,15,20$ and $25 \mu \mathrm{g} / \mathrm{mL}$ each $)$ was placed on each parafilm and was covered with another parafilm to make a pouch. The entire experiment was done in triplicate. Another experiment was set up with a range of different doses of $\mathrm{BjD}(0,10,20,30,40$ and $50 \mu \mathrm{g} / \mathrm{mL}$ each). Water was used as a no diet control to show that the provided liquid diet (without RiD and $\mathrm{BjD}$ ) in comparison with water, can support the survival of the aphids. Aphid survivability was then recorded at every $12 \mathrm{~h}$ interval. Abbot's formula was used to calculate the corrected mortality (Abbott 1925). Statistical Probit analysis using the $X^{2}$ method (Chi 1997) was performed to calculate the $\mathrm{LC}_{50}$ value of $\mathrm{RiD}$ and $\mathrm{BjD}$ against $L$. erysimi. Statistical significance level used was $0.05(\alpha \leq 0.05)$.

\section{Additional files}

Additional file 1: Figure S1. Representative images of $R$. indica and $B$. juncea infested with L. erysimi.

Additional file 2: Fig. S2. MALDI-TOF/TOF-MS spectra of peptides generated from tryptically digested RiD.

Additional file 3: Fig. S3. SignalP-NN Prediction plot, and representation of the RiD: YFP fusion protein.

Additional file 4: Table S1. Sequence of the all the primers used.

\section{Abbreviations}

BjD: Brassica juncea defensin; dpi: day(s) post-infestation; ER: endoplasmic reticulum; GFP: green fluorescent protein; hpi: hour(s) post-infestation; HCCA: a-cyano-4-hydroxycinnamic acid; IPTG: isopropyl $\beta$-D-1-thiogalactopyranoside; $\mathrm{LC}_{50}$ : lethal concentration which causes $50 \%$ mortality of test population; RiD: Rorippa indica defensin; MALDI-TOF/TOF: matrix-assisted laser desorption/ionization time-of-flight; Ni-NTA: nickle-nitrilotriacetic acid; PAGE: polyacrylamide gel electrophoresis; SDS: sodium dodecyl sulfate; TFA: trifluoroacetic acid; YFP: yellow fluorescent protein.

\section{Authors' contributions}

PS and SS conceived and designed the experiments; PS performed the experiments; PS, JJ and SC interpreted the homology modelling data; PS and SS analyzed the data; PS wrote the manuscript. All authors read and approved the final manuscript.

\section{Author details \\ ${ }^{1}$ Division of Plant Biology, Centenary Campus, Bose Institute, Kolkata 700054, India. ${ }^{2}$ Department of Biophysics, Centenary Campus, Bose Institute, Kol-} kata 700054 , India.

\section{Acknowledgements}

The present work was supported by Department of Science and Technology. PS acknowledges Bose Institute for fellowship and for infrastructural facilities. The authors acknowledge Prof. Sampa Das for allowing to use the Real-Time PCR Detection System, Dr. Shubho Chaudhuri for providing the CD3-683 vector and Prof. D.N. Sengupta for allowing to use the particle gene gun for bombardment. PS is also thankful to Mr. Asim Kumar Poddar and Mr. Amarendra Nath Biswas for their technical help in operating the confocal microscope and MALDI-TOF/TOF-mass spectrometer respectively. 


\section{Competing interests}

The authors declare that they have no competing interests.

Received: 3 December 2015 Accepted: 12 April 2016

Published online: 23 April 2016

\section{References}

Abbott WS (1925) A method of computing the effectiveness of insecticide. J Econ Entomol 18:265-267

Abraham V, Bhatia CR (1994) Testing for tolerance to aphids in Indian mustard, Brassica juncea (L.) Czern and Coss. Plant Breed 112:260-263

Altschul SF, Gish W, Miller W, Myers EW, Lipman DJ (1990) Basic local alignment search tool. J Mol Biol 215:403-410

Atri C, Bharti K, Hitesh K, Sarwan K, Sanjula S, Surinder SB (2012) Development and characterization of Brassica juncea-fruticulosa introgression lines exhibiting resistance to mustard aphid (Lipaphis erysimi Kalt). BMC Genet 13:104

Baker NA, Sept D, Joseph S, Holst MJ, McCammon JA (2001) Electrostatics of nanosystems: application to microtubules and the ribosome. Proc Natl Acad Sci 98:10037-10041

Bakhetia DRC, Sekhon BS (1989) Insect pests and their management in rapeseed mustard. J Oilseeds Res 6:269-299

Bandopadhyay S, Roy A, Das S (2001) Binding of garlic (Allium sativum) leaf lectin to the gut receptors of homopteran pest is correlated to its insecticidal activity. Plant Sci 161:1025-1033

Bandopadhyay L, Basu D, Sikdar SR (2013) Identification of genes involved in wild crucifer Rorippa indica resistance response on mustard aphid Lipaphis erysimi challenge. PLoS ONE 8:e73632

Banerjee S, Hess D, Mazumdar P, Roy D, Das S (2004) The interaction of Allium sativum leaf agglutinin with a chaperonin group of unique receptor protein isolated from a bacteria endosymbiont of the mustard aphid. J Biol Chem 279:23782-23789

Banerjee N, Sengupta S, Roy A, Ghosh P, Das K, Das S (2011) Functional alteration of a dimeric insecticidal lectin to a monomeric antifungal protein correlated to its oligomeric status. PLoS ONE 6:e18593

Bloch CJ, Richardson M (1991) A new family of small (5 kDa) protein inhibitors of insect a-amylase from seeds of sorghum (Sorghum bicolour (L.) Moench) have sequence homologies with wheat gamma-thionins. FEBS 279:101-104

Burge C, Karlin S (1997) Prediction of complete gene structures in human genomic DNA. J Mol Biol 268:78-94

Carvalho AO, Gomes VM (2009) Plant defensins-prospects for the biological functions and biotechnological properties. Peptides 30:1007-1020

Carvalho AO, Gomes VM (2011) Plant defensins and defensin-like peptidesbiological activities and biotechnological applications. Curr Pharm Des 17:4270-4293

Chen MS (2008) Inducible direct plant defence against insect herbivores: a review. Insect Sci 15:101-114

Chen KC, Lin CY, Kuan CC, Sung HY, Chen CS (2002) A novel defensin encoded by a mungbean CDNA exhibits insecticidal activity against bruchid. J Agric Food Chem 50:7258-7263

Chi H (1997) Computer program for the probit analysis. National Chung Hsing University, Taichung

Choi MS, Kim YH, Park HM, Seo BY, Jung JK, Kim ST, Kim MC, Shin DB, Yun HT, Choi IS, Kim CK, Lee JY (2009) Expression of Br D1, a plant defensin from Brassica rapa, confers resistance against brown planthopper (Nilaparvata lugens) in transgenic rices. Mol Cells 28:131-137

Dadd R, Mittler T (1976) Permanent culture of an aphid on a totally synthetic diet. Cell Mol Life Sci 22:832-833

Das A, Roy A, Hess D, Das S (2013) Characterization of a highly potent insecticidal lectin from Colocasia esculenta tuber and cloning of its coding sequence. Am J Plant Sci 4:408-416

Dutta I, Majumdar P, Saha P, Ray K, Das S (2005a) Constitutive and phloem specific expression of Allium sativum leaf agglutinin (ASAL) to engineer aphid (Lipaphis erysimi) resistance in transgenic Indian mustard (Brassica juncea). Plant Sci 169:996-1007

Dutta I, Saha P, Majumdar P, Sarkar A, Chakraborti D, Banerjee S, Das S (2005b) The efficacy of a novel insecticidal protein, Allium sativum leaf lectin
(ASAL), against homopteran insects monitored in transgenic tobacco. Plant Biotechnol J 3:601-611

Franco OL (2011) Peptide promiscuity: an evolutionary concept for plant defense. FEBS Lett 585:995-1000

Goomber TS, Labana KS (1983) Development of aphid resistance lines in Brassica juncea (L.). In: 6th international conference on rapeseed COLZA, Paris

Harrison RL, Bonning BC (2010) Proteases as insecticidal agents. Toxins 2:935-953

Harrison SJ, Marcus JP, Goulter KC, Green JL, Maclean DJ, Manners JM (1997) An antimicrobial peptide from the Australian native Hardenbergia violacea provides the first functionally characterized member of a subfamily of plant defensins. Aust J Plant Physiol 24:571-578

Higo K, Ugawa Y, Iwamoto M, Higo H (1998) PLACE: a database of plant cisacting regulatory DNA elements. Nucl Acids Res 26:358-359

Jacobson MP, Pincus DL, Rapp CS, Day TJ, Honig B, Shaw DE et al (2004) A hierarchical approach to all atom protein loop prediction. Proteins 55:351-367

Jaouannet M, Rodriguez PA, Thorpe P, Lenoir CJG, MacLeod R, Martinez CE, Bos JIB (2014) Plant immunity in plant-aphid interactions. Front Plant Sci 5:663

Jong WW, Zweers A, Cohen LH (1978) Influence of single amino acid substitutions on electrophoretic mobility of sodium dodecyl sulfate-protein complexes. Biochem Biophys Res Commun 82:532-539

Jorgensen WL, Maxwell DS, Tirado RJ (1996) Development and testing of the OPLS all-atom force field on conformational energetics and properties of organic liquids. J Am Chem Soc 118:11225-11236

Kanrar S, Venkateswari J, Kirti PB, Chopra VL (2002) Transgenic Indian mustard (Brassica juncea) with resistance to the mustard aphid (Lipaphis erysimi Kalt.). Plant Cell Rep 20:976-981

Kaur J, Thokala M, Seilaniantz AR, Zhao P, Peyret H, Berg H, Pandey S, Jones J, Shah D (2012) Subcellular targeting of an evolutionarily conserved plant defensin MtDef4.2 determines the outcome of plant-pathogen interaction in transgenic Arabidopsis. Mol Plant Pathol 13:1032-1046

Koike M, Okamoto T, Tsuda S, Imai R (2002) A novel plant defensin-like gene of winter wheat is specifically induced during cold acclimation. Biochem Biophys Res Commun 298:46-53

Kragh KM, Nielsen JE, Nielsen KK, Dreboldt S, Mikkelsen JD (1995) Characterization and localization of new antifungal cysteine-rich proteins from Beta vulgaris. Mol Plant Microbe Interact 8:424-434

Kular JS, Kumar S (2011) Quantification of avoidable yield losses in oilseed brassica caused by insect pests. J Plant Prot Res 51:38-43

Kushmerick C, de Souza Castro M, Santos CJ, Bloch CJ, Beirao PS (1998) Functional and structural features of gamma-zeathionins, a new class of sodium channel blockers. FEBS Lett 440:302-306

Lacerda AF, Vasconcelos EAR, Pelegrini PB, Grossi de Sa MF (2014) Antifungal defensins and their role in plant defense. Front Microbiol 5:116

Lawo NC, Wackers FL, Romeis J (2009) Indian Bt cotton varieties do not affect the performance of cotton aphids. PLoS ONE 4:e4804

Lay FT, Anderson MA (2005) Defensins-components of the innate immune system in plants. Curr Protein Pept Sci 6:85-101

Lay FT, Brugliera F, Anderson MA (2003) Isolation and properties of floral defensins from ornamental tobacco and petunia. Plant Physiol 131:1283-1293

Lay FT, Poon S, McKenna JA, Connelly AA, Barbeta BL, McGinness BS, Fox JL, Daly NL, Craik DJ, Heath RL, Anderson MA (2014) The C-terminal propeptide of a plant defensin confers cytoprotective and subcellular targeting functions. BMC Plant Biol 14:41

Lescot M, Dehais P, Moreau Y, De Moor B, Rouze P, Rombauts S (2002) PlantCARE: a database of plant cis-acting regulatory elements and a portal to tools for in silico analysis of promoter sequences. Nucleic Acids Res 30:325-327

Lin KF, Lee TR, Tsai PH, Hsu MP, Chen CS, Lyu PC (2007) Structure based protein engineering for a-amylase inhibitory activity of plant defensin. Proteins Struct Funct Bioinform 68:530-540

Liu YJ, Cheng CS, Lai SM, Hsu MP, Chen CS, Lyu PC (2006) Solution structure of the plant defensin $\mathrm{VrD1}$ from mung bean and its possible role in insecticidal activity against bruchids. Proteins 63:777-786

Livak KJ, Schmittgen TD (2001) Analysis of relative gene expression data using real-time quantitative PCR and the $2^{-\Delta \Delta C t}$ method. Methods 25:402-408

Mandal P, Sikdar SR (2003) Plant regeneration from mesophyll protoplasts of Rorippa indica (L.) Hiern, a wild crucifer. Curr Sci 85:1451-1454 
Mann M (2006) Functional and quantitative proteomics using SILAC. Nat Rev 7:952-958

Melo FR, Rigden DJ, Franco OL, Mello LV, Ary MB, Grossi de Sa MF, Bloch C Jr (2002) Inhibition of trypsin by cowpea thionin: characterization, molecular modelling, and docking. Proteins 48:311-319

Mondal HA, Maiti MK, Basu A, Sen S, Ghosh AK, Sen SK (2006) Transgenic expression of onion leaf lectin in Indian mustard offers protection against aphid colonization. Crop Sci 46:2022-2032

Mondal HA, Roy A, Gupta S, Das S (2012) Exploring the insecticidal potentiality of Amorphophallus paeonifolius tuber agglutinin in hemipteran pest management. Am J Plant Sci 3:780-790

Moran P, Thompson GA (2001) Molecular responses to aphid feeding in Arabidopsis in relation to plant defense pathways. Plant Physiol 125:1074-1085

Moran PJ, Cheng Y, Cassell JL, Thompson GA (2002) Gene expression profiling of Arabidopsis thaliana in compatible plant-aphid interactions. Arch Insect Biochem Physiol 51:182-203

Oomen RJFJ, Carpentier ES, Ricodeau N, Bournaud C, Conejero G, Paris N, Berthomieu P, Marques L (2011) Plant defensin AhPDF1.1 is not secreted in leaves but it accumulates in intracellular compartments. New Phytol 192:140-150

Pelegrini PB, Franco OL (2005) Plant gamma-thionins: novel insights on the mechanism of action of a multi-functional class of defense proteins. Int J Biochem Cell Biol 37:2239-2253

Pelegrini PB, Lay FT, Murad AM, Anderson MA, Franco OL (2008) Novel insights on the mechanism of action of a-amylase inhibitors from the plant defensin family. Proteins 73:719-729

Petersen TN, Brunak S, Heijne G, Nielsen H (2011) SignalP 4.0: discriminating signal peptides from transmembrane regions. Nat Methods 8:785-786

Porcar M, Grenier AM, Federici B, Rahbe Y (2009) Effects of Bacillus thuringiensis delta-endotoxins on the pea aphid (Acrythosiphon pisum). Appl Environ Microbiol 75:4897-7900

Rahbe Y, Febvay G (1993) Protein toxicity to aphids: an in vitro test on Acyrthosiphon pisum. Entomol Exp Appl 67:149-160

Rahbe Y, Deraison C, Bonade-Bottino M, Girard C, Nardon C, Jouanin L (2003) Effects of the cysteine protease inhibitor oryzacystatin (OC-I) on different aphids and reduced performance of Myzus persicae on OC-I expressing transgenic oilseed rape. Plant Sci 164:441-450
Rath A, Glibowickaa M, Nadeaua VG, Chena G, Debera CM (2009) Detergent binding explains anomalous SDS-PAGE migration of membrane proteins. Proc Natl Acad Sci 106:1760-1765

Robert X, Gouet P (2014) Deciphering key features in protein structures with the new ENDscript server. Nucl Acids Res 42:320-324

Roy A, Gupta S, Hess D, Das KP, Das S (2014) Binding of insecticidal lectin Colocasia esculenta tuber agglutinin (CEA) to midgut receptors of Bemisia tabaci and Lipaphis erysimi provides clues to its insecticidal potential. Proteomics 14:1646-1659

Santos IS, Carvalho AO, Filho GA, Nascimento VV, Machado OLT, Gomes VM (2010) Purification of a defensin isolated from Vigna unquiculata seeds, its functional expression in Escherichia coli, and assessment of its insect a-amylase inhibitory activity. Protein Expr Purif 71:8-15

Shaner NC, Steinbach PA, Tsien RY (2005) A guide to choosing fluorescent proteins. Nat Methods 2:905-909

Singh RN, Das R, Saran G, Singh RK (1982) Differential response of mustard varieties to aphid, Lipaphis erysimi (Kalt.). Indian J Entomol 44:408

Terras F, Schoofs H, De Bolle M, Van Leuven F, Rees S, Vanderleyden J, Cammue B, Broekaert W (1992) Analysis of two novel classes of plant antifungal proteins from radish (Raphanus sativus L.) seeds. J Biol Chem 267:15301-15309

Terras F, Torrekens S, Van Leuven F, Osborn R, Vanderleyden J, Cammue B, Broekaert W (1993) A new family of basic cysteine-rich plant antifungal proteins from Brassicaceae species. FEBS Lett 316:233-240

Thompson JD, Higgins DG, Gibson TJ (1994) CLUSTALW: improving the sensitivity of progressive multiple sequence alignment through sequence weighing, position-specific gap penalties and weight matrix choice. Nucleic Acids Res 22:4673-4680

Verma SN, Singh OP (1987) Estimation of avoidable losses to mustard by the aphid, Lipaphis erysimi (Kalt.) in Madhya Pradesh. Indian J Plant Prot 15:87-89

Vriens K, Cammune BPA, Thevissen K (2014) Antifungal plant defensins: mechanisms of action and production. Molecules 19:12280-12303

Will T, Vilcinskas A (2015) The structural sheath protein of aphids is required for phloem feeding. Insect Biochem Mol Biol 57:34-40

Zhang Y, Lewis K (1997) Fabatins: new antimicrobial plant peptides. FEMS Microbiol Lett 149:59-64

\section{Submit your manuscript to a SpringerOpen ${ }^{\circ}$ journal and benefit from:}

- Convenient online submission

- Rigorous peer review

- Immediate publication on acceptance

- Open access: articles freely available online

- High visibility within the field

- Retaining the copyright to your article

Submit your next manuscript at $\boldsymbol{\nabla}$ springeropen.com 